\title{
Moderhinke beim Schaf -
neue Ansätze zur Sanierung
}

\author{
Deborah Greber
}

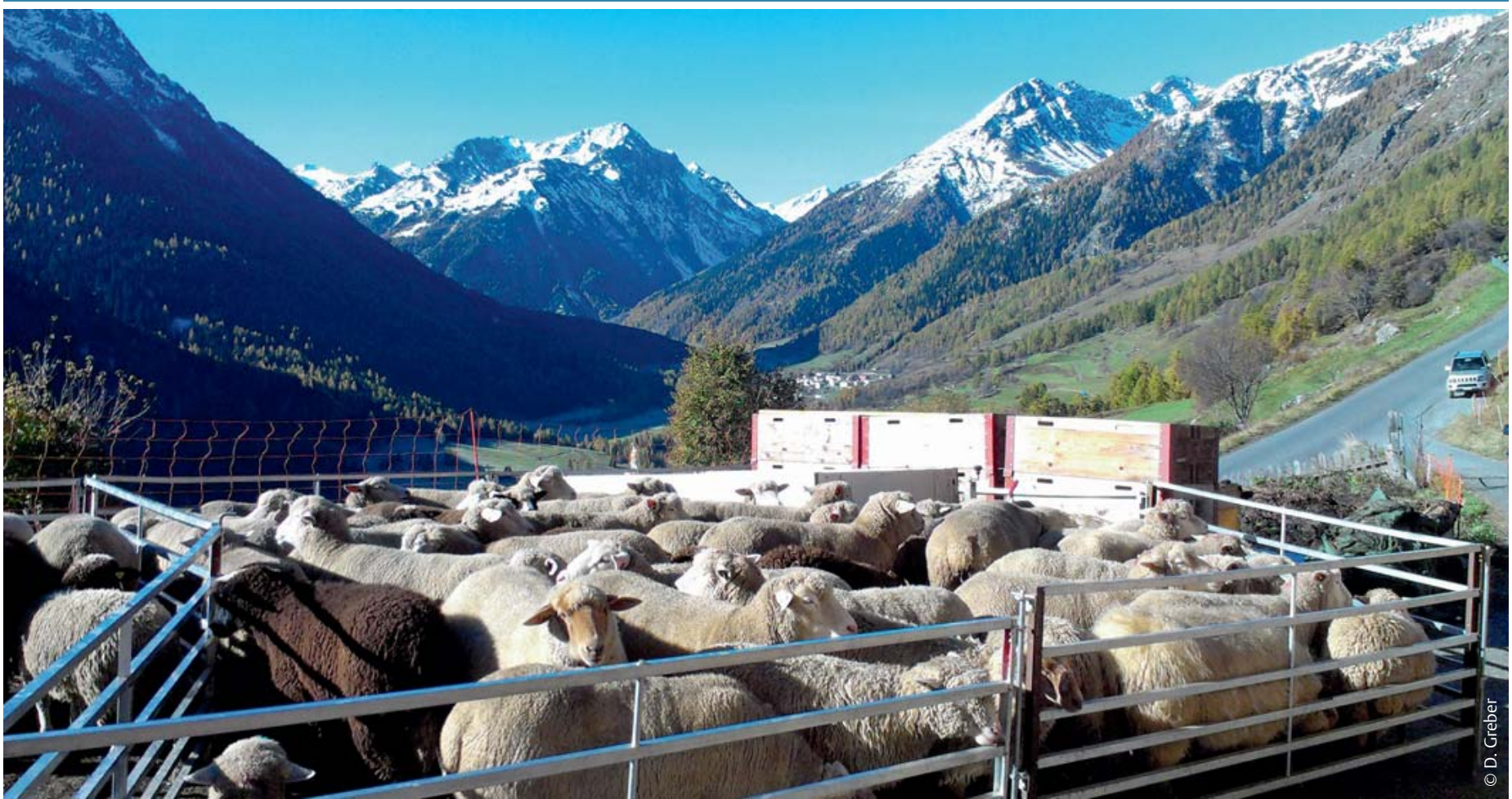

Die Moderhinke ist ein intensiv diskutiertes Thema, zu dem es verschiedenste Ansätze für Therapie und Bekämpfung gibt. Mit neuen diagnostischen Mitteln ist es nun möglich, den Erreger zuverlässig nachzuweisen und den Weg für eine nachhaltige Bekämpfung der Moderhinke frei zu machen. Wir stellen Ihnen ein aktuelles Bekämpfungsschema vor, unterstützt durch neue Laboruntersuchungen, mit regelmäßigen Klauenbädern und konsequenter Ausmerzung und Isolierung.

\section{Fakten}

Die Moderhinke (Klauenfäule) ist eine ansteckende und schmerzhafte Klauenkrankheit kleiner Wiederkäuer, die weltweit vorkommt und alle Schafrassen betrifft. Sie ist die häufigste Ursache für Lahmheit bei
Schafen und führt zu erheblichen wirtschaftlichen Einbußen, wie z.B. Verlusten in der Wollproduktion, verminderter Mastleistung sowie dem damit einhergehenden Zeitaufwand zur Behandlung der befallenen Tiere.

Die Krankheit wird verursacht durch das Bakterium Dichelobacter nodosus, das obligat vorhanden sein muss, in Zusammenhang mit dem sekundären ubiquitären Erreger Fusobacterium necrophorum. Der Einfluss und die Rolle von Fusobacterium necrophorum wird in der Literatur jedoch kontrovers diskutiert. Dichelobacter (D.) nodosus ist ein gramnegatives, obligat anaerobes Bakterium, das vor allem im Klauenhorn überlebt, durch kleine Läsionen in der Epidermis im Zwischenklauenspalt eindringt und sich dann unter Luftabschluss vermehren kann.

\section{Dichelobacter nodosus}

D. nodosus weist verschiedene VirulenzFaktoren auf, wie z. B. Proteasen, die ein Auflösen des Klauenhorns ermöglichen $[1,4]$. Die verschiedenen Proteasen werden unter anderem kodiert durch die Gene AprV2, AprV5 und BprV (für die virulenten Enzyme) und die Gene AprB2, AprB5 und BprB (für die gutartigen Enzyme). In der Studie von Stäuble et al. 2014 wurde nachgewiesen, dass die Enzyme AprB2 und AprV2 gut mit der Klinik korrelieren, wobei AprV2 nachweislich für die virulente klinische Form von Moderhinke verantwortlich ist $[1,7]$.

Untersuchungen in Australien im Jahr 1941 zeigten, dass der Erreger außerhalb des Klauenhorns in Mist und Schlamm 7 Tage bis 2 Wochen überleben kann, doch Trockenheit, Hitze und Kälte $\left(<10^{\circ} \mathrm{C}\right)$ redu- 


\section{Moderhinkescore 0 bis 5}

Score 0: gesunde Klaue mit trockenem Zwischenklauenspalt ( $\odot$ Abb. 1)

Score 1: Zwischenklauenspalt (ZKS) feucht, gerötet, mit Haarausfall

Score 2: ausgebreitete Entzündung im ZKS mit schmierigem Belag, leicht geschädigtes Horn am Rand der inneren Klauenwand

Score 3: deutlich schmierige Beläge im ZKS und verändertes Gewebe unterhalb der inneren Klauenwand und des Sohlenhorns (ه Abb. 2)

Score 4: ausgedehnte Loslösung des Hornes über die Sohle bis zur äußeren Wand der Klaue; das darunterliegende Gewebe ist stark geschädigt

Score 5: „Ausschuhen“: Loslösen des kompletten Klauenhorns von der Klaue

zieren die Überlebenszeit des Bakteriums in der Umwelt erheblich [8]. In neueren Untersuchungen in einem feuchten Gebiet mit regelmäßigen Niederschlägen (Mitteleuropa) wurde der Erreger auch noch nach 14 Tagen mittels PCR in der Umgebung nachgewiesen. Da das Experiment nach 14 Tagen endete, bleibt unklar, wie lange der Erreger bei optimalen Bedingungen noch überleben würde [1].

Mittels PCR kann das Vorhandensein von Genmaterieal von D. nodosus in der Umwelt zwar besser und genauer untersucht werden als bisher, doch wie lange die maximale Überlebenszeit des Erregers in hiesigen klimatischen Bedingungen tatsächlich ist, ist nach wie vor unbekannt.

Man geht davon aus, dass $D$. nodosus in abgeschnittenem Klauenhornmaterial Monate überleben kann.

Aus diesem Grund wird dringend empfohlen, den Klauenschnitt an einem trockenen, befestigten Platz durchzuführen und nach dem Klauenschnitt alles abgeschnittene Klauenmaterial zusammenzukehren und zu verbrennen.

\section{Diagnose und Symptome}

Die Diagnose der Moderhinke wird vor allem anhand des klinischen Bildes gestellt,

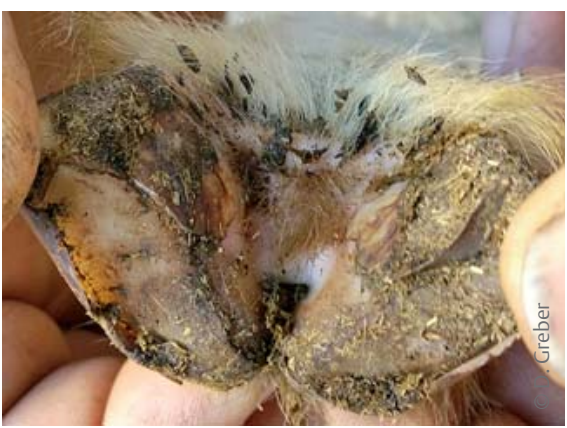

Abb. 1 Klaue mit Moderhinkescore 0: Eine gesunde Klaue mit trockenem Zwischenklauenspalt.

da es bis vor kurzem keine geeigneten labordiagnostischen Möglichkeiten für den Nachweis gab. Das Bakterium mittels einer bakteriologischen Untersuchung anzuzüchten, ist aufgrund seines anspruchsvollen Wachstumsverhaltens oft schwierig oder gar erfolglos.

Das klinische Bild der Krankheit reicht von einer

- benignen Form (ausgelöst durch benigne Stämme mit dem AprB2-Enzym), die sich in einer leichteren Rötung im Zwischenklauenspalt zeigt, bis zu einer

- schweren virulenten Form (ausgelöst durch virulente Stämme mit dem AprV2-Enzym), bei der es zur kompletten Unterminierung des Klauenhorns und zum Ausschuhen kommen kann.

In Regionen, in denen die Moderhinke endemisch vorkommt, fressen befallene Schafe oft charakteristischerweise zur Entlastung auf den Carpi, da die damit einhergehenden entzündlichen Veränderungen an den Klauen zu massiven Schmerzen führen. Mit zunehmendem Bewusstsein und Aufklärung der Bevölkerung in Hinblick auf Tierwohl und Tierschutz wächst der Druck auf die Tierhalter, denn inzwischen ist vielen bewusst, dass „kniend“ grasende Schafe nicht niedlich sind, sondern dass sie ihre Klauen entlasten, weil sie Schmerzen haben.

\section{Management}

Es ist seit über 60 Jahren bekannt, dass Herdensanierungen bei einem Moderhinkebefall möglich sind. Die Bekämpfung der

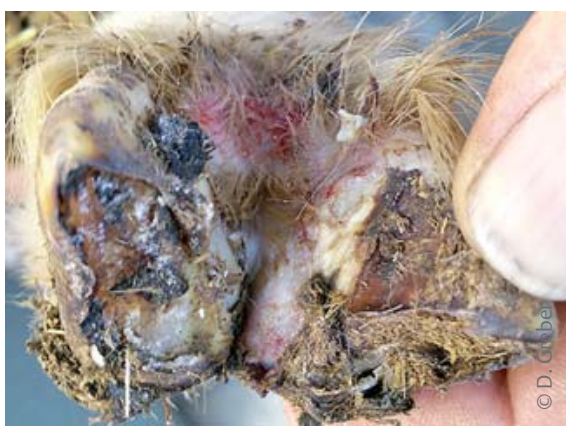

Abb. 2 Klaue mit Moderhinkescore 3: Deutliche schmierige Beläge im Klauenzwischenbereich und loslösen des Klauenhorns der Innenwand.

Klinik von Moderhinke auf Herdenebene wurde mittels Vakzinen, Antibiotika, desinfizierenden Klauenbädern und anderen Methoden schon erfolgreich durchgeführt. Oft sind erfolgreiche klinische Herdensanierungen jedoch überschattet von Reinfektionen resp. Wiederaufflammen der klinischen Symptome. Dies führt über eine längere Zeit dazu, dass sich Schafhalter mit der Krankheit arrangieren und lediglich versuchen, den Schaden in Grenzen zu halten.

Sanierungen müssen konsequent durchgeführt werden, wobei es auf das Zusammenspiel verschiedener Maßnahmen als „Paket" ankommt:

- Alle Tiere müssen durch ein Klauenbad laufen ( Abb. 3).

- Der Stall muss gereinigt werden, um Neuinfektionen zu vermeiden.

- Die Tiere sind auf eine neue Weide zu führen.

- Alle Tiere einer Herde sollen behandelt werden, auch solche, die vermeintlich gesunde Klauen haben. Gerade diese Schafe können subklinische Träger sein und später für Reinfektionen sorgen $[2,4]$.

\section{Schweizer Forschung}

In der Schweiz wurde in den letzten 10 Jahren vermehrt zum Thema Moderhinke geforscht und in verschiedenen Projektgruppen wurden mehrere Themen wissenschaftlich erarbeitet. Die Ergebnisse dienten als Grundlage für eine im Frühjahr 2014 an das Schweizer Parlament gestellte Motion (Beauftragung des Schweizer Bundesrats, innerhalb von 2 Jahren einen Entwurf für 


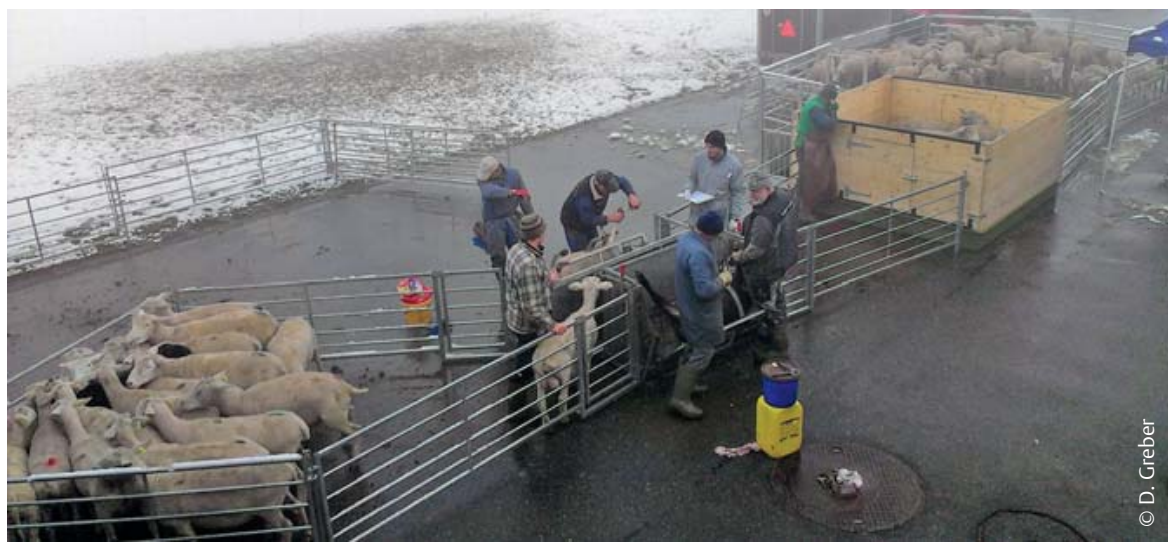

Abb. 3 Aufbau eines Wartebereichs vor dem Klauenbad. Die gesamte Behandlung wird auf befestigtem Boden durchgeführt, da sich dieser besser reinigen lässt. Das gesamte infizierte Klauenmaterial muss anschließend im Abfall entsorgt werden.

ein Gesetz oder einen Beschluss vorzulegen oder eine Maßnahme zu treffen) für eine staatlich vorgeschriebene schweizweite Bekämpfung der Moderhinke beim Schaf.

Bisher wurde in der Schweiz ein Sanierungsschema durchgeführt, das auf einer Herdentrennung anhand des klinischen $\mathrm{Zu}$ stands der Klauen basierte. Dabei wurde die Herde in eine gesunde und eine kranke Gruppe aufgeteilt und die kranke Gruppe wöchentlich mit einem Klauenbad in Zinksulfat, Kupfersulfat oder Formaldehyd behandelt. Die gesunde Gruppe wurde getrennt von der kranken gehalten und alle 2 Wochen gebadet. Wenn die Tiere in der kranken Gruppe klinisch gesund waren, wurden sie in die gesunde Gruppe verbracht. Hierbei gab es immer wieder Rückschläge und durch die subjektive Beurteilung der Klauen auch unterschiedliche Meinungen, ob es sich um ein beginnendes Stadium der Moderhinke handelt oder doch nur um eine leichte Entzündung anderer Genese.

\section{Neues Sanierungsschema}

Nun wurde von Greber et al. (2016) ein neues Sanierungsschema getestet, bestehend aus gezieltem Klauenschnitt, wöchentlichem Klauenbad, Kontrollen, die auf PCR-Diagnostik basierten, und der Ausmerzung von latent infizierten Tiere [2].

Dies gelang, weil in der Schweiz in den letzten Jahren an der Wiederkäuerklinik und dem Institut für Veterinärbakteriologie der Vetsuisse-Fakultät der Universität Bern eine PCR etabliert wurde, die in der Lage ist, zwischen virulenten und benignen Stämmen anhand der obengenannten Enzyme AprV2 und AprB2 von $\boldsymbol{D}$. nodosus zu unterscheiden [7]. Somit kann objektiv unterschieden werden, ob die Rötung im Zwischenklauenspalt von einer beginnenden Moderhinke-Infektion kommt oder evtl. andere harmlose Ursachen hat. Zusätzlich können auf diesem Wege symptomfreie Träger von $D$. nodosus identifiziert werden.

Es wurde bei diesem neuen Sanierungsschema bewusst auf den Einsatz von Antibiotika verzichtet, da der Einsatz von Antibiotika in der Veterinärmedizin aufgrund der Resistenzproblematik heute sehr kritisch beurteilt wird. Auch auf eine Impfung wurde verzichtet, da sie nicht alle Stämme abdeckt und zudem nur zu einer geringen vorübergehenden klinischen Besserung führt, den Erreger jedoch nicht abtötet. In der an der Wiederkäuerklinik der Vetsuisse-Fakultät der Universität Bern durchgeführten Sanierungsstudie von Greber et al. (2016) wurden insgesamt 28 Schafherden erfolgreich saniert [2]. Die klinischen Untersuchungen wurden von Herbst 2014 bis Frühling 2015 durchgeführt.

\section{Durchführung}

Das neue Sanierungsschema basiert auf dem wöchentlichen Klauenbad in einer Zinksulfat-Lösung, Kontrolle durch PCR, vorsichtigem Klauenschnitt und Ausmerzung oder Isolation von therapieresistenten und somit latent infizierten Schafen.
Dabei wurde folgendermaßen vorgegangen:

1. Beim 1. Besuch in Woche 1 wurden alle Tiere überprüft und von jedem Schaf ein Klauenscoring nach BGK (Beratung und Gesundheitsdienst Kleinwiederkäuer Schweiz; Tabelle 1: Score 1-5) durchgeführt.

2. Von maximal 5 Tieren wurde ein Tupfer entnommen, um zu verifizieren, dass es sich um den virulenten Stamm des D. nodosus handelt.

3. Anschließend wurde ein sorgfältiger Klauenschnitt durchgeführt.

4. Nach der Kontrolle wurden alle Tiere für 10 Minuten in ein Zinksulfat Klauenbad verbracht. Danach wurde pro Woche ein 10-minütiges Klauenbad durch die Besitzer durchgeführt.

Cave: Die Anwendung von Zinksulfat-Lösungen für Klauenbäder in Deutschland ist zumeist nur bei einem Therapienotstand möglich. Vor der Verwendung sollte das zuständige Veterinäramt dazu kontaktiert werden.

Die Besitzer wurden vor dem Beginn der Studie mit einem Informationsschreiben versorgt und auf die wichtigsten Hygienemaßnahmen hingewiesen.

In Woche 4 fand ein 2. Kontrollbesuch statt. Dabei wurden alle Tiere, bei denen in der ersten Kontrolle ein Klauenscore $>2$ festgestellt wurde, nochmals kontrolliert und ein erneuter Klauenscore durchgeführt. Der Kontrollbesuch diente als Motivationsstütze für die Tierhalter und als Kontrolle, ob die Klauenbäder korrekt durchgeführt wurden.

In Woche 7 wurde ein Kontrollbesuch mit Scoring, Tupferprobennahme und PCR durchgeführt. Bei Herden, in denen mindestens ein Tier einen Klauenscore von $>2$ und somit deutliche klinische Veränderungen hatte, wurden maximal 5 Tupfer ausgewertet, um zu überprüfen, ob es sich immer noch um den virulenten Stamm mit dem AprV2-Gen des D. nodosus handelte. Bei den Herden ohne klinisch positive Tiere wurden alle Tupferproben ausgewertet. Falls alle Proben aprV2-negativ waren, wurde die Gruppe als erfolgreich saniert betrachtet. 


\section{Positive Restbestände}

Wenn jedoch immer noch aprV2-positive Tiere darin waren, wurde die ganze Herde wöchentlich weitergebadet und nach 3-4 Wochen nochmals kontrolliert, bis alle Tiere aprV2-negativ waren. Falls es sich um Einzeltiere handelte, die noch positiv waren, wurde den Tierhaltern geraten, diese Schafe auszumerzen oder zu isolieren und nur noch die isolierten Tiere weiter zu baden und kontrollieren zu lassen. Bei allen Betrieben wurde im darauffolgenden Frühling eine Nachkontrolle durchgeführt. Bei der Nachkontrolle wurden alle Klauen aller Schafe kontrolliert und von jedem Tier eine Tupfer-Probe für die PCR-Untersuchung entnommen.

\section{Erfolgreiche Sanierung}

Es wurde in insgesamt 33 Herden (im Schnitt 56 Tiere) mit der Sanierung angefangen, wobei 2 Herden ausgeschlossen wurden, weil sie keine Schafe mit klinischen Zeichen von Moderhinke hatten. Weitere 3 Herden wurden nach 3, 6 und 8 Wochen ausgeschlossen, weil die Halter nicht dem vorgegebenen Sanierungsschema gefolgt waren. Die Herden umfassten im Schnitt 56 Tiere, wobei der kleinste Betrieb 11 und der größte Betrieb 111 Tiere umfasste.

Beim ersten Kontrollbesuch in Woche 7 waren 7 Betriebe erfolgreich saniert, das heißt, es waren alle Klauentupfer aprV2negativ. Wenn der Erfolg nur anhand der klinischen Beurteilung gemessen worden wäre, wären in Woche 716 der 28 Betriebe als klinisch saniert klassifiziert worden. Somit wiesen 9 der 16 Betriebe Schafe auf, die klinisch gesund waren, jedoch immer noch DNA-Material vom $D$. nodosus aufwiesen und eventuell potenzielle Reinfektionsquellen wären.

In Woche 9 waren 14 von 29 Betrieben erfolgreich saniert. Beim ersten Besuch variierte die klinische Prävalenz von Moderhinke zwischen 6,3-79,6\% (median= 38,9\%). Der Wert des durchschnittlichen Klauenscores innerhalb der Herde korrelierte stark mit der Anzahl Klauenbäder, die bis zu einer erfolgreichen Sanierung durchgeführt werden mussten. Zwischen
Take Home: Herdensanierung bei Moderhinke

Die Schlussfolgerung aus der Studie Greber et al. (2016) ist, dass eine Herdensanierung in Form einer vollständigen Elimination des virulenten Erregers D. nodosus aus den Klauen der Schafe mit konsequenter und regelmäßiger Durchführung von desinfizierenden Klauenbädern, vorsichtigem Klauenschnitt und Ausmerzung oder strikter Isolation von Einzeltieren, die ungenügend auf die Behandlung ansprechen, möglich ist.

der Herdengröße und der Dauer der Sanierung wurde kein Zusammenhang gefunden.

Bei den Frühlingskontrollen waren alle 28 Betriebe klinisch und aprV2-negativ. Einer der 28 Betriebe hatte jedoch Tiere, die aprB2-positiv getestet wurden. Dies liegt vermutlich daran, dass der Betrieb die Schafe über den Winter in einem gemeinsamen Auslauf mit Hochlandrindern hielt. In der Literatur wurde schon mehrmals nachgewiesen, dass Rinder Träger des benignen Enzyms AprB2 sein können.

\section{Nationales Konzept}

In der Schweiz wird nun eine Informationskampagne geführt, im Zuge derer im Jahr 2017 mehrere Artikel in verschiedenen Fachzeitschriften erscheinen, um die Tierhalter und die Bevölkerung für die Moderhinke zu sensibilisieren. Gleichzeitig wird in mehreren Arbeitsgruppen, bestehend aus Expertinnen und Experten verschiedener Fachgebiete und Schafhaltenden ein nationales Bekämpfungskonzept entwickelt.

Eine der großen Herausforderungen eines nationalen Bekämpfungskonzeptes ist es, die unterschiedlichen Ausgangslagen von verschiedenen Haltungsformen $\mathrm{zu}$ berücksichtigen und den hohen Tierverkehr von Schafen in der Schweiz unter Kontrolle zu bekommen. Der Beginn einer nationalen Bekämpfung der Moderhinke wird nicht vor 2019 erwartet [6]. Gleichzeitig laufen an der Vetsuisse-Fakultät der Universität Bern noch weitere wissenschaftliche Studien zum Erreger D. nodosus. Es wird unter anderem die Überlebensfähigkeit von $D$. nodosus auf verschiedenen Vektoren untersucht, wie Klauenmesser, Kleider, Transporter etc., um das Übertragungspotenzial bei der Klauenpflege und Klauenschur besser beurteilen zu können.

\section{Literatur}

1 Cederlöf SE, Hansen T, Klaas IC et al. An evaluation of the ability of Dichelobacter nodosus to survive in soil. Acta Vet Scand 2013; 55: 4

2 Greber D, Bearth G, Lüchinger R et al. Elimination of virulent strains (aprV2) of Dichelobacter nodosus from feet of 28 Swiss sheep flocks: A proof of concept study. Vet J 2016; 216: 25-32

3 Kennan, R.M., Wong, W., Dhungyel, O.P. et al. The subtilisin-like protease AprV2 is required for virulence and uses a novel disulphidetethered exosite to bind substrates. PLoS Pathogens 6 (e01210).

4 Locher I, Greber D, Holdener K et al. Longitudinal Dichelobacter nodosus status in 9 sheep flocks free from clinical footrot. Small Rumin Res 2015; 132: 128-132

5 Palmer MA. A gelatin test to detect activity and stability of proteases produced by Dichelobacter (Bacteroides) nodosus. Vet Microbiol 1993; 36: 113-122

6 Schweizerische Eidgenossenschaft Bundesamt für Lebensmittelsicherheit und Veterinärwesen. Bekämpfung Moderhinke (Projekt). Online im Internet: https://www.blv.admin. ch/blv/de/home/tiere/tierseuchen/ bekaempfung/projekt-moderhinke.html, Stand: 28.04.2017

7 Stäuble A., Steiner A., Frey J et al. Simultaneous detection and discrimination of virulent and benign Dichelobacter nodosus in sheep of flocks affected by foot rot and in clinically healthy flocks by competitive realtime PCR. J Clin Microbiol 2014; 52: 12281231

8 Whittington RJ. Observations on the indirect transmission of virulent ovine footrot in sheep yards and its spread in sheep on unimproved pasture. Aust Vet J 1995, 72: 132-134

\section{Online}

https://doi.org/10.1055/s-0043-116456

\section{Verfasserin}

Dr. Deborah Greber

Tierklinik und tierärztliche Praxis Dr. Schneichel Ostbahnhofstr. 77

56727 Mayen

deborah.greber@gmx.ch 